\section{Microscopy}

Coming Events

\section{9}

Advanced Microscopy Techniques for Plant-microbe Interaction Analysis November 25-29, 2019

Tulln, Austria

www. plant-microbe-microscopy.com/program

\section{MRS 2019 Fall Meeting}

December 1-6, 2019

Boston, MA

www.mrs.org/fall2019

\section{ASCB 2019 Annual Meeting}

December 7-11, 2019

Washington, CA

www.ascb.org/meetings-events/future-ascbmeetings

\section{APMC-2020 - 12th Asia-Pacific}

Microscopy Conference

February 3-7, 2020

Hyderabad, India

www.apmc12.in

Biophysical Society $64^{\text {th }}$ Annual Meeting

February 15-19, 2020

San Diego, CA

www.biophysics.org/Meetings/AnnualMeeting/

FutureAnnualMeetings/tabid/495/Default.aspx

ACMM 26 - 26th Australian

Conference on Microscopy and

Microanalysis

February 15-20, 2020

Canberra, Australia

www.acmm26.org/welcome

\section{0}

Microscopy \& Microanalysis 2020

August 2-6, 2020

Milwaukee, WI

www.microscopy.org

\section{1}

Microscopy \& Microanalysis 2021

August 1-5, 2021

Pittsburgh, PA

www.microscopy.org

\section{2}

Microscopy \& Microanalysis 2022

July 31-August 4, 2022

Portland, OR

www.microscopy.org

\section{3}

Microscopy \& Microanalysis 2023

July 24-28, 2023

Minneapolis, MN

www.microscopy.org

\section{4}

Microscopy \& Microanalysis 2024

July 28-August 1, 2024

Cleveland, $\mathrm{OH}$

www.microscopy.org

More Meetings and Courses

Check the complete calendar near the back of this magazine.

\title{
It Can Bite You Even if You Can't See It
}

\author{
Stephen W. Carmichael \\ Mayo Clinic, Rochester, MN 55905 \\ carmichael.stephen@mayo.edu
}

Dragonfish (Aristostomias scintillans) are apex predators of the deep sea. Because interactions of these fish with humans are so rare, they do not capture the same attention as apex predators of the shallow waters, such as great white sharks and pirahanas. Dragonfish typically feed on relatively plentiful lantern fish and bristlemouths, which are thought to be one of the most abundant vertebrates in nature. The dragonfish lures the smaller fish with a light-producing barbel, a beard-like structure attached to its chin. The mouth with spearlike teeth must remain hidden for effective hunting. The creature's translucent teeth are key to this camouflage (Figure 1). What makes the teeth almost transparent was recently revealed by Audrey Velasco-Hogan, Dimitri Deheyn, Marcus Koch, Birgit Nothdurft, Eduard Artz, and Marc Meyers [1].

Several different methods were used in this study, including light microscopy, hyperspectral imaging (to quantify transparency of the teeth), micro-computed tomography, transmission electron microscopy (TEM), scanning electron microscopy (SEM), and environmental SEM, which allows examination of wet specimens by using specialized electron detectors and other modifications. Using these and other methods, Velasco-Hogan et al. showed that teeth of the dragonfish are composed of two homogeneous concentric layers, an enamel-like one and dentin, which constitute the walls of the teeth with the enamel on the outer surface. Importantly, the teeth have a nanostructure that reduces Rayleigh scattering of light. These teeth lack the larger microscale features, such as dentin tubules, thus light scattering is

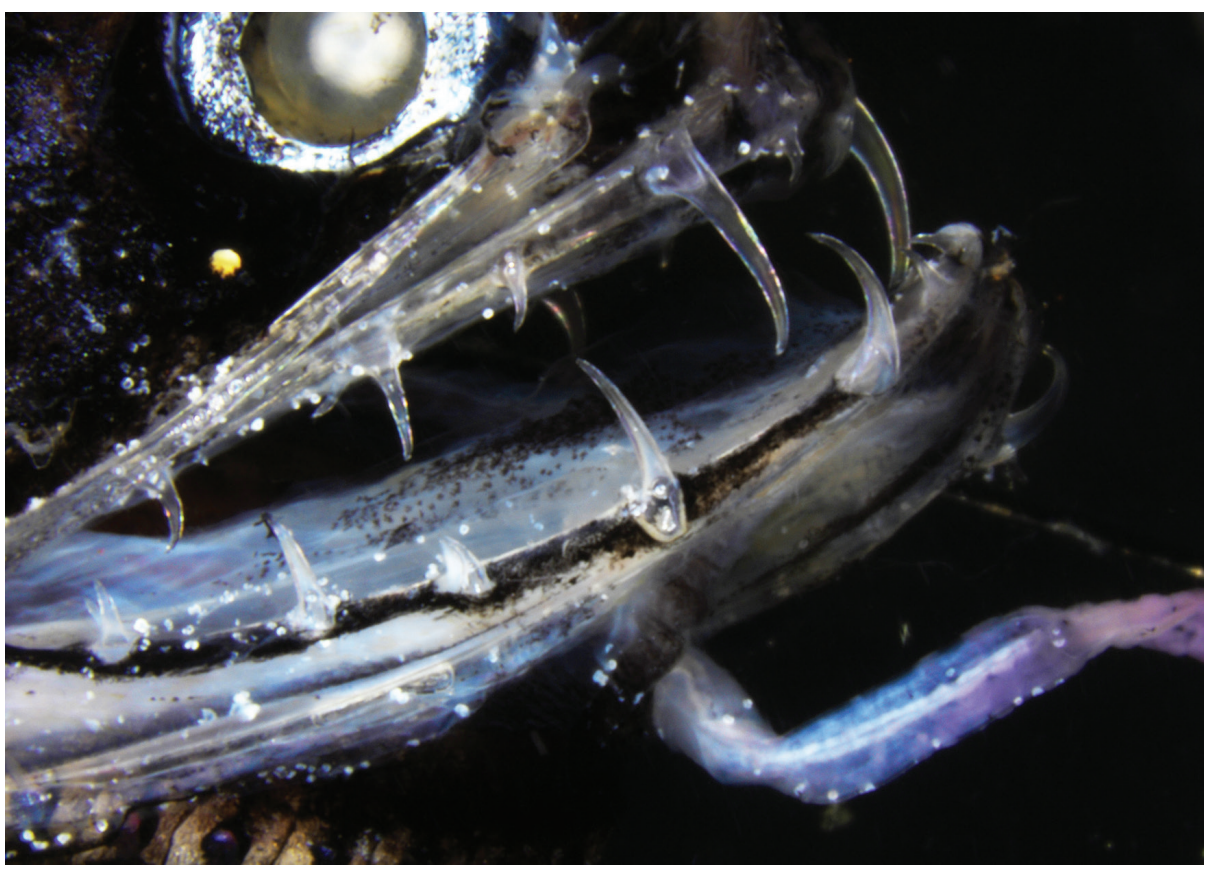

Figure 1: The dragonfish has a black body and nearly transparent teeth, which make this deep-sea fish an efficient predator. Image width $=20 \mathrm{~mm}$. 


\section{A Plasma FIB-SEM platform}

\section{for deep sectioning and the highest}

\section{resolution end-pointing}

\section{for package level failure analysis}

J)

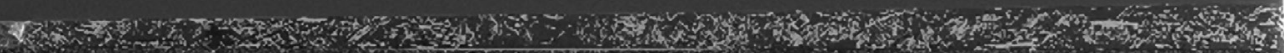

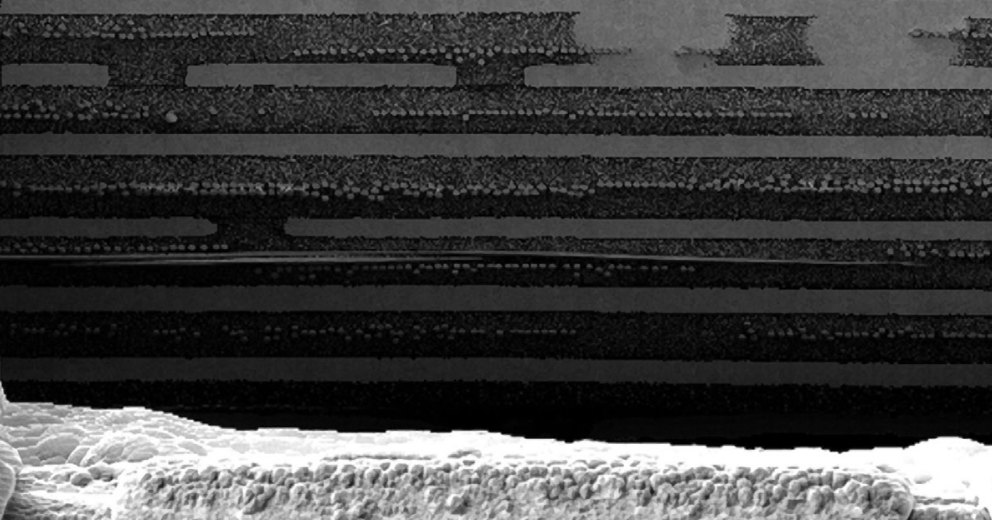

\section{TESCAN SOLARIS X}

$\checkmark$ Curtaining-free large-area cross-sectioning for physical failure analysis of advanced packaging technologies.

$\checkmark$ Prepare large area FIB-cross-sections up to $1 \mathrm{~mm}$ wide.

$\checkmark$ Obtain low noise, high resolution image at low keVs in short acquisition time at FIB-SEM coincidence with the sample tilted.

$\checkmark$ Live SEM-monitoring during FIB milling for precise end-pointing.

$\checkmark$ Observe the most beam-sensitive materials using low keVs ultra-high resolution for surface sensitivity and high material contrast.

$\checkmark$ Effective techniques and recipes for fast and artefact-free cross-sectioning of composite samples (OLED and TFT displays, MEMS devices, isolation dielectrics) at high currents.

$\checkmark$ Essence $^{\text {TM }}$ easy-to-use modular user interface

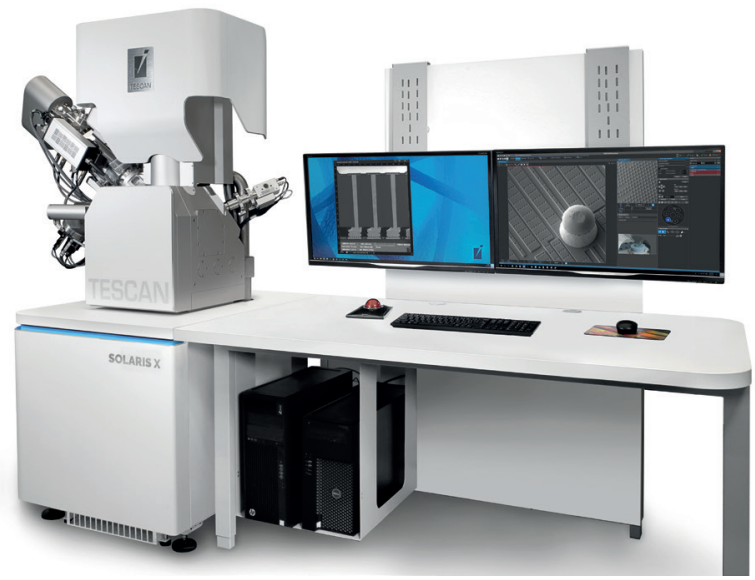

For more information visit 
reduced and transparency is increased. Normally particle scattering would make the scattering medium more visible, however nanosize particles cause reduced scattering effectively making the tooth less visible.

Atomic-resolution TEM on specimens thinned to about $40 \mathrm{~nm}$ shows the characteristics of both the enamel-like and dentin layers. The enamel-like layer consists of individual crystallites embedded in an amorphous matrix. The dentin structure consists of very small rods (about $5 \mathrm{~nm}$ in diameter) forming an interwoven pattern. Study of electron diffraction patterns of the teeth suggested that the crystalline inorganic material is hydroxyapatite, the main inorganic component found in teeth and bone. The presence of carbon indicated collagen as the organic component. This was confirmed using Fourier transform infrared spectroscopy. The size of the particles is consistent with the observed reduction in Rayleigh scattering.

The hardness and other properties of the teeth were measured using a nanoindenter. The hardness of the enamel was found to be comparable with the teeth of the great white shark and piranha. Despite the significant differences in size, morphology, and habitat, the mechanical properties of the enamel-like layer of the three predatory species were strikingly similar. When compared to the dentin of the great white shark and piranha, the dragonfish dentin was much harder. This suggested that it has a higher degree of mineralization and nanoscale structural arrangement, both of which should contribute to transparency.

Velasco-Hogan et al. showed that the teeth of dragonfish owe their transparency to a nanoscale structure of hydroxyapatite and collagen that lacks larger microscale features such as dentin tubules, which are a feature of teeth in most animals. The structure is consistent with mechanical strength and reduced Rayleigh scattering that makes the teeth strong yet almost transparent. A variety of characterization techniques demonstrated that the teeth are extremely hard and sharp, being comparable with those from great white sharks and piranhas. This new finding correlates well with the fact that the dragonfish is an efficient predator. The authors have shown for the first time how the hard sharp teeth of the dragonfish represents a deadly invisible weapon for an efficient predator [2].

\section{References}

[1] A Velasco-Hogan et al., Aristostomias scintillans, Matter 1 (2019) 1-15.

[2] The author gratefully acknowledges Dr. Marc Meyers for reviewing this article.
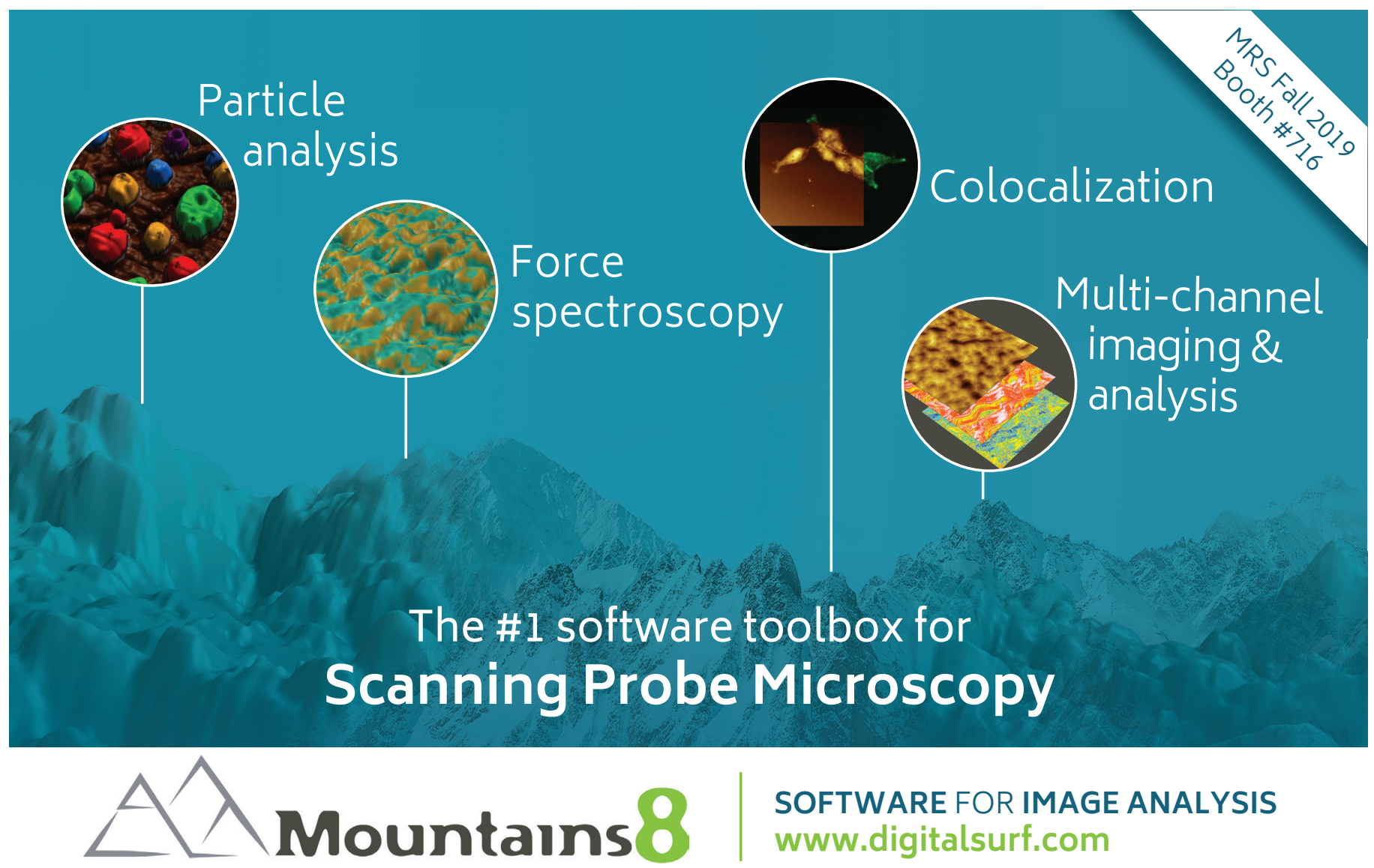

SOFTWARE FOR IMAGE ANALYSIS Www.digitalsurf.com 


\section{Electron}

Microscopy sciences

Pictured: Neurons from rat embryonic dorsal root ganglion.

FlowVIEW Aquarius "Liquid" Scanning EM Kit

K-Kit Wet "Liquid" TEM Kit

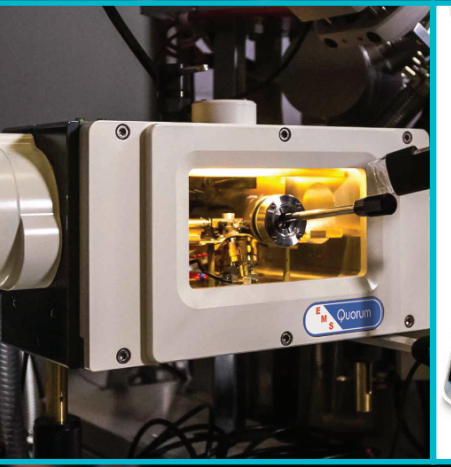

Quorum PP3010 Cryo-SEM/Cryo-FIB/SEM Preparation System
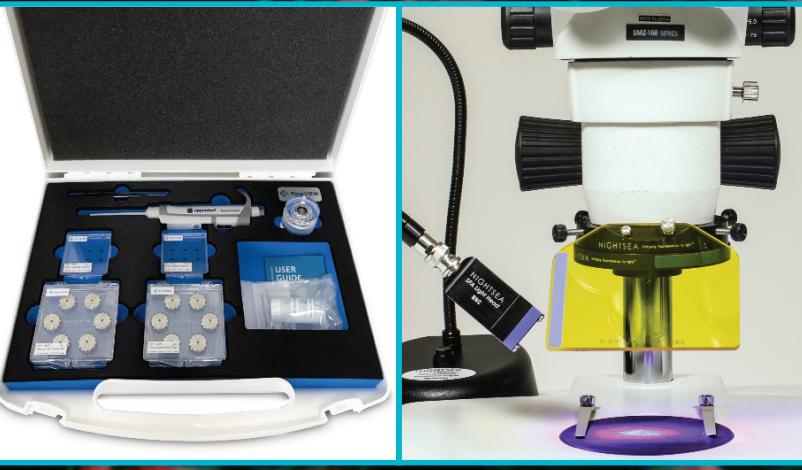

NIGHTSEA

Stereo Microscope

Fluorescence Adapter
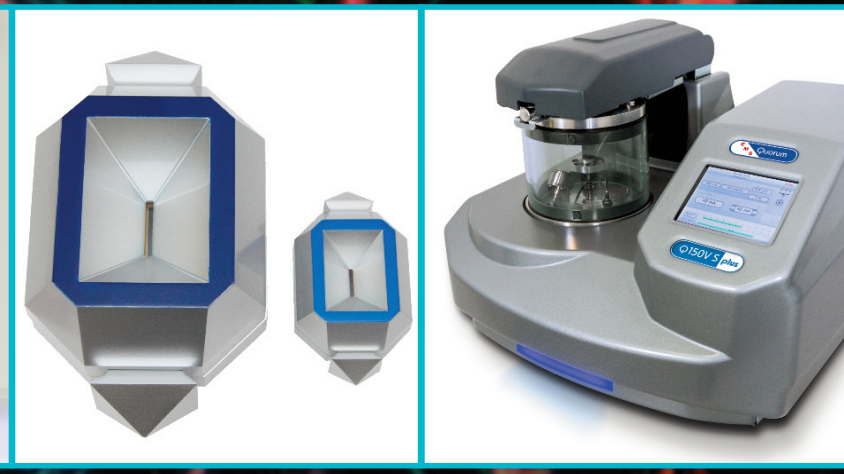

Quorum Q150V Plus for ultra fine coatings

\section{your comprehensive source}

\section{for all fields of microscopy and general laboratory research}

ELECTRON MICROSCOPY

LIGHT MICROSCOPY

HISTOLOGY SUPPLIES

CHEMICALS

VACUUM EQUIPMENT

and more.

\section{www.emsdiasum.com}

Electron Microscopy Sciences

P.0. Box 550 - 1560 Industry Rd. • Hatfield, PA 19440

Tel: (215) 412-8400 • Faxt (215) 412-8450

email: info@emsdiasum.com or stacie@ems-secure.com 